\title{
On the Detection of Supermassive Primordial Stars
}

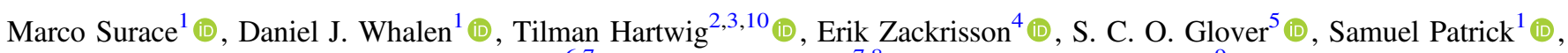 \\ Tyrone E. Woods ${ }^{6,7}$ (D), Alexander Heger ${ }^{7,8}$ (iD), and Lionel Haemmerlé ${ }^{9}$ \\ ${ }^{1}$ Institute of Cosmology and Gravitation, University of Portsmouth, Portsmouth PO1 3FX, UK; daniel.whalen@port.ac.uk \\ ${ }^{2}$ Department of Physics, School of Science, University of Tokyo, Bunkyo, Tokyo 113-0033, Japan \\ ${ }^{3}$ Kavli IPMU (WPI), UTIAS, The University of Tokyo, Kashiwa, Chiba 277-8583, Japan \\ ${ }^{4}$ Department of Physics and Astronomy, Uppsala University, Box 516, SE-751 20 Uppsala, Sweden \\ ${ }^{5}$ Universität Heidelberg, Institut für Theoretische Astrophysik, Albert-Ueberle-Str. 2, D-69120 Heidelberg, Germany \\ ${ }^{6}$ Institute of Gravitational Wave Astronomy and School of Physics and Astronomy, University of Birmingham, Birmingham B15 2TT, UK \\ ${ }^{7}$ Monash Centre for Astrophysics, School of Physics and Astronomy, Monash University, VIC 3800, Australia \\ 8 Tsung-Dao Lee Institute, Shanghai 200240, People's Republic of China \\ ${ }^{9}$ Observatoire de Genève, Université de Genève, chemin des Maillettes 51, CH-1290 Sauverny, Switzerland \\ Received 2018 November 14; revised 2018 December 4; accepted 2018 December 12; published 2018 December 21
}

\begin{abstract}
The collapse of supermassive primordial stars in hot, atomically cooled halos may have given birth to the first quasars at $z \sim 15-20$. Recent numerical simulations of these rapidly accreting stars reveal that they are cool, red hypergiants shrouded by dense envelopes of pristine atomically cooled gas at 6000-8000 K, with luminosities $L \gtrsim 10^{10} L_{\odot}$. Could such luminous but cool objects be detected as the first stage of quasar formation in future nearinfrared (NIR) surveys? We have now calculated the spectra of supermassive primordial stars in their birth envelopes with the Cloudy code. We find that some of these stars will be visible to the James Webb Space Telescope at $z \lesssim 20$ and that with modest gravitational lensing, Euclid and the Wide-Field Infrared Space Telescope could detect them out to $z \sim 10-12$. Rather than obscuring the star, its accretion envelope enhances its visibility in the NIR today by reprocessing its short-wavelength flux into photons that are just redward of the Lyman limit in the rest frame of the star.
\end{abstract}

Key words: black hole physics - dark ages, reionization, first stars - early universe - galaxies: formation galaxies: high-redshift - quasars: general

\section{Introduction}

Supermassive primordial stars (SMSs) may have been the origin of the first quasars, a few of which have now been discovered at $z>7$ (e.g., Mortlock et al. 2011; Bañados et al. 2018). These stars are thought to form in primordial halos exposed to either unusually strong Lyman-Werner (LW) UV fluxes (Agarwal et al. 2016) or highly supersonic baryon streaming motions (Hirano et al. 2017; Schauer et al. 2017). Either one can prevent primordial halos from forming stars until they reach masses of $10^{7}-10^{8} M_{\odot}$ and virial temperatures of $\sim 10^{4} \mathrm{~K}$ that trigger rapid atomic cooling and catastrophic baryon collapse at central infall rates of up to $\sim 1 M_{\odot} \mathrm{yr}^{-1}$ (Bromm \& Loeb 2003; Lodato \& Natarajan 2006; Wise et al. 2008; Regan \& Haehnelt 2009; Latif et al. 2013).

Stellar evolution models show that Population III stars growing at these rates can reach masses of a few $10^{5} M_{\odot}$. Most then collapse to black holes (direct collapse black holes, or DCBHs; Umeda et al. 2016; Woods et al. 2017; Haemmerlé et al. 2018a) via the general relativistic (GR) instability; although, a few non-accreting stars have been found to explode as highly energetic thermonuclear transients (Johnson et al. 2013a; Chen et al. 2014; Whalen et al. 2013a, 2013b, 2014). Population III SMSs are currently the leading contenders for the seeds of the earliest supermassive black holes (SMBHs) because the environments of ordinary Population III star BHs are less conducive to their rapid growth (Whalen et al. 2004; Alvarez et al. 2009; Whalen \& Fryer 2012; Smith et al. 2018). DCBHs are born with large masses in high densities in host

\footnotetext{
${ }^{10}$ JSPS Fellow.
}

galaxies that can retain their fuel supply, even when they are heated by X-rays (Johnson et al. 2013b).

What are the prospects for detecting SMSs at high redshifts? Hartwig et al. (2018) found that the relics of such stars would be uniquely identifiable with the gravitational wave detector LISA at $z>15$ if they form in binaries. Johnson et al. (2012) examined some spectral features of hot, blue, rapidly accreting SMSs and found that they would be characterized by strong Balmer emission and the conspicuous absence of Ly $\alpha$ lines due to absorption by their envelopes. The source of this flux was not the star but its hypercompact $\mathrm{H}$ II region, whose ionizing radiation was trapped close to its surface by the density and ram pressure of the inflow (which was also found to be true in cosmological simulations of highly resolved atomically cooled halos; Becerra et al. 2018). Freese et al. (2010), Zackrisson et al. (2010a, 2010b) calculated the spectral signatures of hot, blue Population III "dark stars", supermassive primordial stars powered by the self-annihilation of weakly interacting dark matter rather than by nuclear fusion. They found that such objects might be visible even to $8-10 \mathrm{~m}$ telescopes on the ground today, primarily because of their high surface temperatures $\left(20000-30000 \mathrm{~K}\right.$ ), larger masses (up to $10^{7} M_{\odot}$ ), and longer lives (up to $10^{7} \mathrm{yr}$ ).

But several studies have now shown that rapidly accreting Population III stars generally evolve as cool, red hypergiants along the Hayashi limit with surface temperatures of 5000-10000 K (Hosokawa et al. 2013). Haemmerlé et al. (2018b) found that such stars can reach luminosities $\gtrsim 10^{10} L_{\odot}$ and could in principle be visible to James Webb Space Telescope (JWST; Kalirai 2018), Euclid, WFIRST, and extremely large telescopes (ELTs) on the ground. However, 

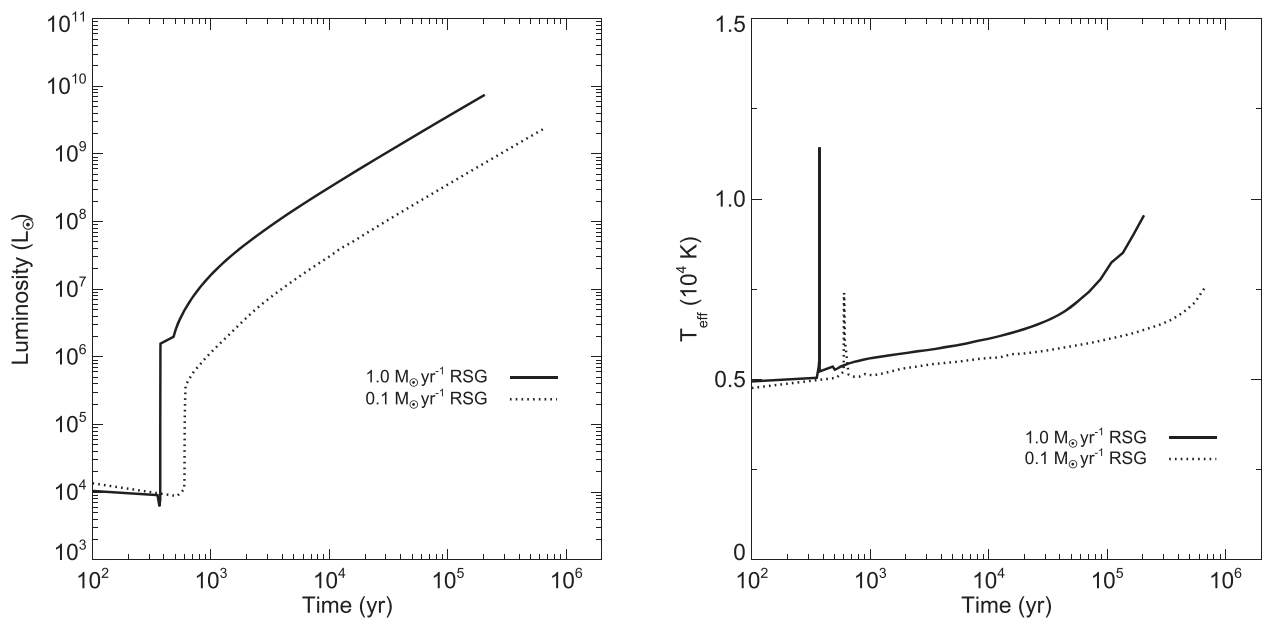

Figure 1. Evolution of red supergiant (RSG) stars accreting at 1.0 and $0.1 M_{\odot} \mathrm{yr}^{-1}$ in the GENEVA stellar evolution code. Left panel: luminosities. Right panel: surface temperatures.

they are shrouded by dense accretion flows that reprocess radiation from the star, perhaps suppressing its flux in the NIR today. Detecting SMSs at high redshift would capture primordial quasars at the earliest stages of their development and reveal one of their channels of formation. Here, we calculate NIR luminosities for Population III SMSs in their accretion envelopes whose structures are taken from a highresolution cosmological simulation. We describe our models in Section 2, calculate SMS spectra and NIR magnitudes in Section 3, and conclude in Section 4.

\section{Numerical Method}

Rest frame spectra for the star in its envelope are calculated with Cloudy (Ferland et al. 2017) with envelope profiles taken from a simulation of the collapse of an atomically cooled halo done with Enzo (Bryan et al. 2014). The spectra are then redshifted, dimmed, and convolved with a variety of filter functions to obtain $\mathrm{AB}$ magnitudes in the NIR as a function of SMS redshift. We consider stars accreting at 0.1 and $1.0 M_{\odot} \mathrm{yr}^{-1}$ whose properties are listed in Tables A3 and A2 of Haemmerlé et al. (2018b), respectively. Bolometric luminosities, $L_{\mathrm{bol}}$, and effective temperatures, $T_{\text {eff }}$, for both stars are shown in Figure 1.

\subsection{Enzo Envelope Model}

The halo in which the SMS is assumed to form was evolved in a $1.5 h^{-1} \mathrm{Mpc}$ box in Enzo from $z=200$ down to $z=17.8$, when it reaches a mass of $2.7 \times 10^{7} M_{\odot}$ and begins to atomically cool and collapse. It is centered in three nested grids for an initial effective resolution of $2048^{3}$, and we allow up to 15 levels of refinement for a maximum resolution of 0.014 pc. The grid is initialized at $z=200$ with cosmological parameters taken from the second-year Planck release: $\Omega_{\mathrm{M}}=0.308$, $\Omega_{\Lambda}=0.691, \Omega_{\mathrm{b}}=0.0223, h=0.677, \sigma_{8}=0.816$, and $n=$ 0.968 (Planck Collaboration et al. 2016). To approximate the presence of a strong LW background, we evolve the halo without $\mathrm{H}_{2}$, just $\mathrm{H}, \mathrm{H}^{+}, \mathrm{e}^{-}, \mathrm{He}, \mathrm{He}^{+}$, and $\mathrm{He}^{++}$(Smith et al. 2017b). Cooling by collisional ionization and excitation of $\mathrm{H}$ and $\mathrm{He}$, bremsstrahlung, and inverse Compton scattering are all included in the energy equation.
As shown in the upper left panel of Figure 2, a large atomically cooled disk forms at the center of the halo that is $\sim 2 \mathrm{pc}$ in diameter and at $4000-6000 \mathrm{~K}$ at $0.625 \mathrm{Myr}$ after the onset of collapse. A bar instability in the disk efficiently transports angular momentum out of its center, producing the large sustained accretion rates onto the star shown in the upper right panel of Figure 2. After a brief burst due to initial collapse and the formation of the disk, infall proceeds at rates of $0.4-0.6 M_{\odot} \mathrm{yr}^{-1}$. Spherically averaged density and temperature profiles of the halo are shown in the bottom two panels of Figure 2 at $0.238,0.506,1.012$, and $1.786 \mathrm{Myr}$.

\subsection{Cloudy Spectra}

We treat both stars as blackbodies (BBs) because they are relatively cool and have no absorption lines due to metals. Cloudy fits BB spectra to each star from $L_{\mathrm{bol}}$ and $T_{\mathrm{eff}}$, which we take to be $1.26 \times 10^{9} L_{\odot}$ and $6653 \mathrm{~K}$ for the $0.1 M_{\odot} \mathrm{yr}^{-1}$ star and $3.92 \times 10^{9} L_{\odot}$ and $8241 \mathrm{~K}$ for the $1.0 M_{\odot} \mathrm{yr}^{-1}$ star. These values correspond to $3.49 \times 10^{5} \mathrm{yr}$ and $1.089 \times 10^{5} \mathrm{yr}$ for the two stars, about halfway through their respective lifetimes. Ideally, one would surround the star with the accretion envelope that created it in a cosmological simulation for selfconsistency. But stellar evolution models of Population III SMSs in time-dependent cosmological flows are not yet available, so we instead use density and temperature profiles from the Enzo simulation at $1.786 \mathrm{Myr}$ as the envelope of each star. This choice is reasonable because the accretion rates associated with these profiles are intermediate to those in which our stars were evolved.

The density and temperature profiles of the envelope that are input to Cloudy are tabulated in 70 bins that are uniformly partitioned in $\log$ radius, with inner and outer boundaries at $0.015 \mathrm{pc}$ and $927 \mathrm{pc}$. Cloudy solves the equations of radiative transfer, statistical and thermal equilibrium, ionization and recombination, and heating and cooling to calculate the excitation and ionization state of the gas surrounding the star and compute its emergent spectrum. The temperatures of the gas falling onto the star are set by the virialization of cosmic flows well above it, not by radiation from the star. Since they determine to what degree the envelope is collisionally excited, and thus how it reprocesses photons from the star, we required Cloudy to use the temperatures Enzo calculates for the 

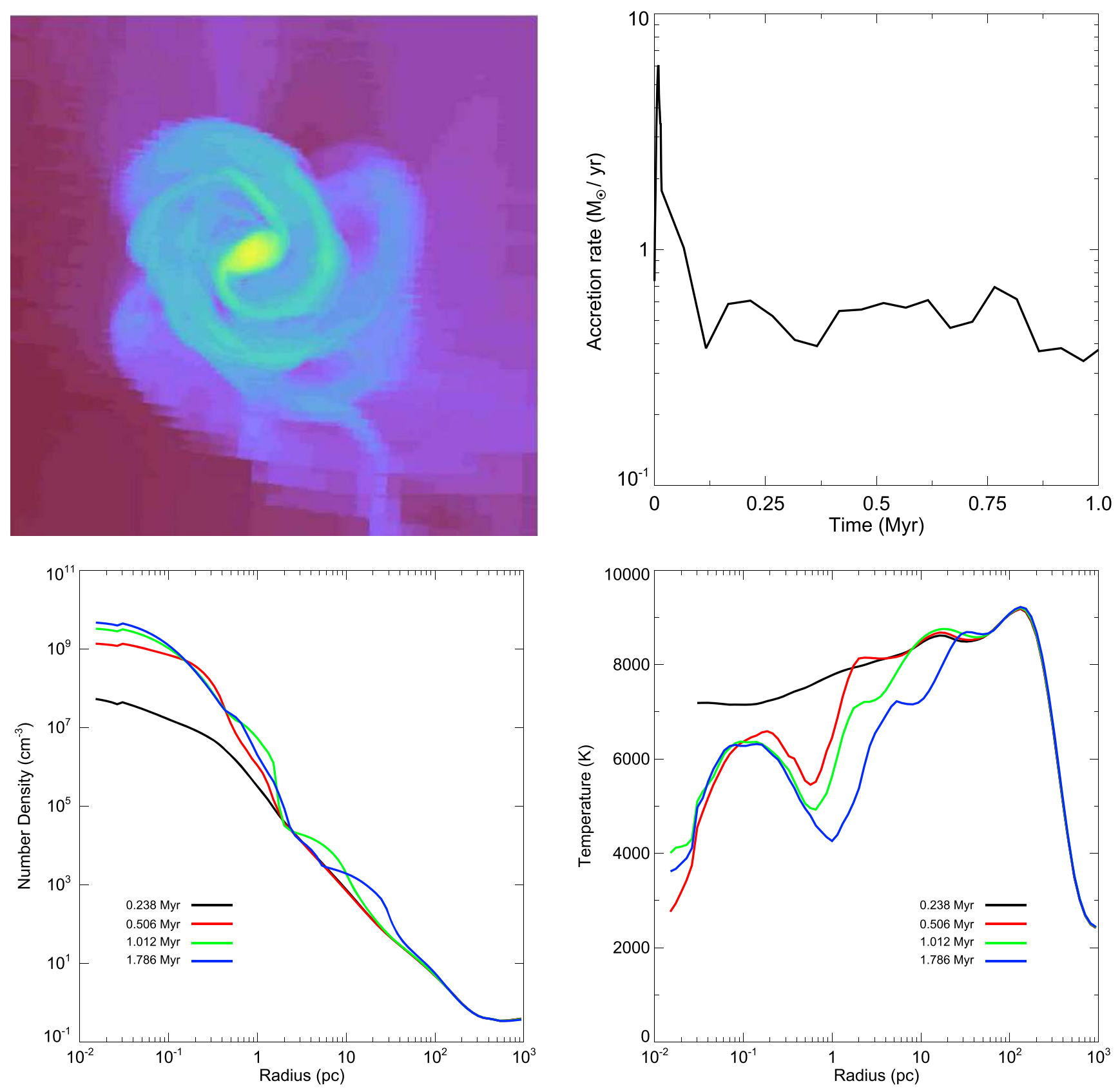

Figure 2. Top left: accretion disk at $0.625 \mathrm{Myr}$. Top right: central accretion rates. Bottom left: spherically averaged gas densities in the halo. Bottom right: spherically averaged temperatures.

envelope instead of inferring them from the spectrum of the SMS because they would have been too low.

\section{Observing Supermassive Stars}

\subsection{SMS Spectra}

We show spectra for the $1.0 M_{\odot} \mathrm{yr}^{-1}$ star at $1.089 \times 10^{5} \mathrm{yr}$ before and after attenuation by its envelope in Figure 3. As expected, the stellar spectrum peaks at $0.352 \mu \mathrm{m}$ and absorption by the envelope at the Lyman limit of $\mathrm{H}$ is clearly visible at $0.0912 \mu \mathrm{m}$. The continuum absorption below $0.0912 \mu \mathrm{m}$ is punctuated by several prominent $\mathrm{He}$ emission lines. There is a Ly $\alpha$ emission line at $0.1216 \mu \mathrm{m}$, and strong $\mathrm{H} \alpha$ and Paschen series lines are visible at $0.656,1.28$, and $1.88 \mu \mathrm{m}$. There is continuum absorption half a decade in wavelength above and below $1.65 \mu \mathrm{m}$ due to $\mathrm{H}^{-}$bound-bound and bound-free opacity, respectively.

Photons from the star that are blueward of the Lyman limit are reprocessed by its envelope into the $\mathrm{Ly} \alpha$ and two-photon continuum emission visible at $0.1216-0.16 \mu \mathrm{m}$. This latter flux is greater than that emitted by the star itself and can enhance its visibility in the NIR today. The effect varies with $T_{\text {eff }}$ and source redshift but is at most $0.5-1 \mathrm{AB}$ mag. The Ly $\alpha$ will not aid in the detection of the star because it will be scattered into a halo of low surface brightness in the neutral IGM. We note that at the velocities and densities of the infall onto the surface of the star, the luminosity of its accretion shock is at most $\sim 10^{4} L_{\odot}$ and does not produce a significant contribution to the visibility of the SMS. 


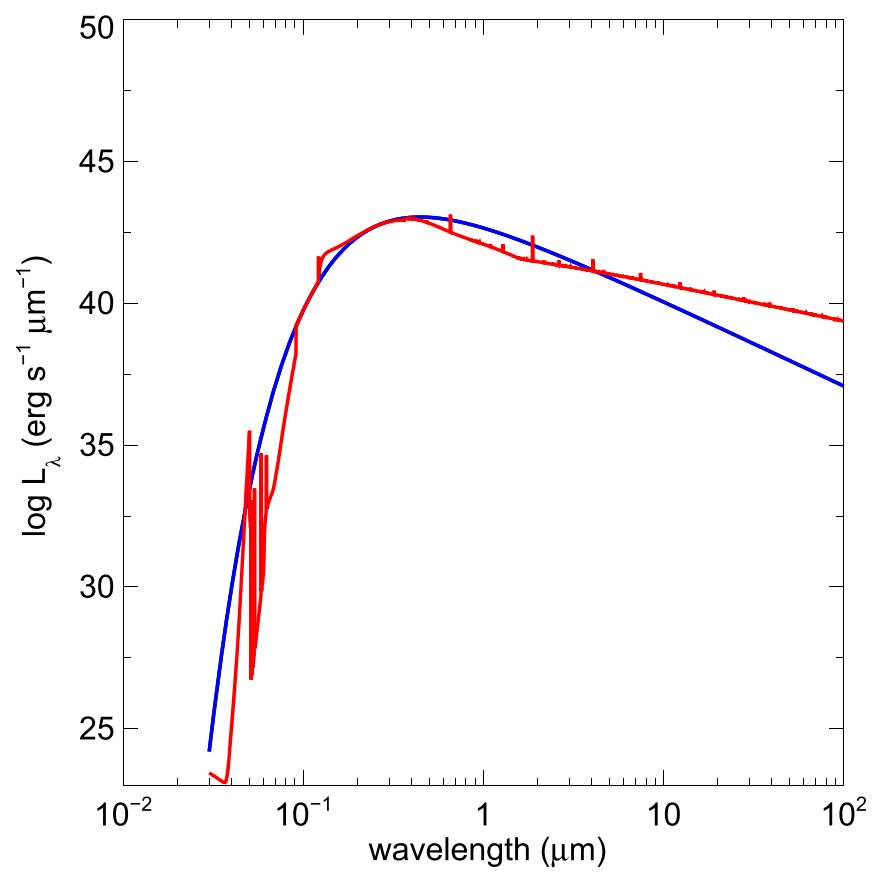

Figure 3. $1.0 M_{\odot} \mathrm{yr}^{-1}$ SMS spectra at 100,000 yr. Blue: spectrum of the star itself; red: spectrum after reprocessing by the envelope.

\subsection{NIR Magnitudes}

We show AB magnitudes for both stars in JWST NIRCam bands at $2.5-4.6 \mu \mathrm{m}$ in the top right panel of Figure 4 . The $1.0 M_{\odot} \mathrm{yr}^{-1} \mathrm{SMS}$ is consistently $1-2 \mathrm{mag}$ brighter than the $0.1 M_{\odot} \mathrm{yr}^{-1} \mathrm{SMS}$ except at high redshifts at $2.50 \mu \mathrm{m}$, where both luminosities abruptly fall off because of absorption of flux blueward of $\operatorname{Ly} \alpha$ in the source frame of the star by the neutral intergalactic medium (IGM) at $z \gtrsim 6$. At $z \sim 6-8$, the two stars are brightest in the $3.56 \mu \mathrm{m}$ filter, but at $z>10$, they are brighter in the 4.44 and $4.60 \mu \mathrm{m}$ bands, with magnitudes that vary from $28.5-31.5$ at $z=6-20$ for the $1.0 M_{\odot} \mathrm{yr}^{-1} \mathrm{SMS}$ and 29.5-33.5 for the $0.1 M_{\odot} \mathrm{yr}^{-1} \mathrm{SMS}$.

SMS magnitudes are much more uniform in redshift in the mid infrared, as we show for several JWST MIRI bands in the top right panel of Figure 4. They exhibit the greatest variation at $5.6 \mu \mathrm{m}$, which is closest to the NIR, but largely level off at $7.7-25.5 \mu \mathrm{m}$. This behavior is primarily due to the flattening of the spectrum at wavelengths above $1.5 \mu \mathrm{m}$ in the source frame due to reprocessing of flux from the star by its envelope. The two stars are brightest from $z=6-20$ at 5.6-10.0 $\mu \mathrm{m}$, with magnitudes $\lesssim 31$ and could therefore provide important additional spectral confirmation of SMS candidates in NIRCam.

We show SMS magnitudes for Euclid and WFIRST in the lower two panels of Figure 4. Absorption by the neutral IGM at $z \gtrsim 6$ quenches $Y, J$, and $H$ band fluxes at $z \gtrsim 7,10$, and 14 , respectively, limiting detections of these stars to these redshifts in these filters. Magnitudes for the $1.0 M_{\odot} \mathrm{yr}^{-1}$ star vary from 29.5-31.8 in Euclid and 29-32.5 in WFIRST at $z=6$. For the $0.1 M_{\odot} \mathrm{yr}^{-1}$ star, they vary from $31.5-32.5$ in Euclid and from 31-32.5 in WFIRST at the same redshift. They drop off more rapidly with redshift than in the NIRCam bands because spectral luminosities in the source frame fall with decreasing wavelength below $\sim 0.3 \mu \mathrm{m}$.

\subsection{SMS Formation/Detection Rates}

Since the lifetime of an SMS is much smaller than the Hubble time, even at the high redshifts at which it is likely to form, the number of SMSs per unit redshift per unit solid angle at a redshift $z$ can be written as

$$
\frac{d N}{d z d \Omega}=\dot{n}_{\mathrm{SMS}} t_{\mathrm{SMS}} r^{2} \frac{d r}{d z},
$$

where $\dot{n}_{\text {SMS }}$ is the SMS formation rate per unit comoving volume, $t_{\mathrm{SMS}}$ is the characteristic lifetime of an SMS, and $r(z)$ is the comoving distance to redshift $z$,

$$
r(z)=\frac{c}{H_{0}} \int_{0}^{z} \frac{d z^{\prime}}{\sqrt{\Omega_{\mathrm{m}}\left(1+z^{\prime}\right)^{3}+\Omega_{\Lambda}}} .
$$

Unfortunately, $\dot{n}_{\mathrm{SMS}}$ is poorly constrained, with theoretical models predicting number densities that vary by up to eight orders of magnitude (see, e.g., the recent review of Woods et al. 2018). These models also predict different evolutions in $\dot{n}_{\mathrm{SMS}}$ with redshift. Habouzit et al. (2016) predict a steady increase in the comoving number density of SMSs with decreasing $z$, while Valiante et al. (2017) predict that most form in the narrow range $z \sim 16-18$. We therefore consider two toy models that bracket this range of $\dot{n}_{\mathrm{SMS}}$.

In the first, our "optimistic" model, we assume that most SMSs form at $z \sim 10-12$ and that the final comoving number density is around $10^{-1} \mathrm{Mpc}^{-3}$, as in the Habouzit et al. (2016) model with a low value for $J_{\text {crit }}$. In the other, our "pessimistic" model, we assume that most SMSs form at redshifts $z \sim 16-18$, as in Valiante et al. (2017), with a final comoving number density of around $10^{-8} \mathrm{Mpc}^{-3}$. The optimistic model yields approximately $4 \times 10^{7}$ potentially observable SMSs per steradian per unit redshift, or around 30 per NIRCam field of view. On the other hand, the pessimistic model predicts only $\sim 10$ SMSs per steradian per unit redshift, meaning that any given NIRCam pointing with the appropriate limiting magnitude would have a probability of only around $10^{-5}$ of detecting an SMS.

The chances of detecting an SMS are highly dependent on the model assumed for their formation. However, since some models predict number counts high enough for one or more SMSs to be found in any sufficiently deep NIRCam image, JWST will begin to place observational constraints on these models, even if it cannot rule out extreme ones such as our pessimistic model. We note that no SMSs have been found in the Hubble Ultra Deep Field to date because of its $\mathrm{AB}$ mag limit of 29 at $1.38 \mu \mathrm{m}$ in the $H$ band, which is well below that expected of either star even at $z \sim 6$.

\section{Conclusion}

At NIRCam AB magnitude limits of 31.5, JWST could detect the $1.0 M_{\odot} \mathrm{yr}^{-1} \mathrm{SMS}$ at $z \lesssim 20$ and the $0.1 M_{\odot} \mathrm{yr}^{-1} \mathrm{SMS}$ at $z \lesssim 13$. But the prospects for discovering such stars would be better if they could also be found by Euclid and WFIRST because their wide fields would enclose far more SMSs at high redshifts. Once flagged, SMS candidates could then be studied with JWST in greater detail. However, as shown in Figure 4, the $H$ band magnitudes of both stars at $z=6-20$ are above current Euclid and WFIRST detection limits (26 and 28, respectively).

But this does not mean Euclid and WFIRST will not find these stars, because only modest gravitational lensing is required to boost their fluxes above the detection limits. The fields of view of 

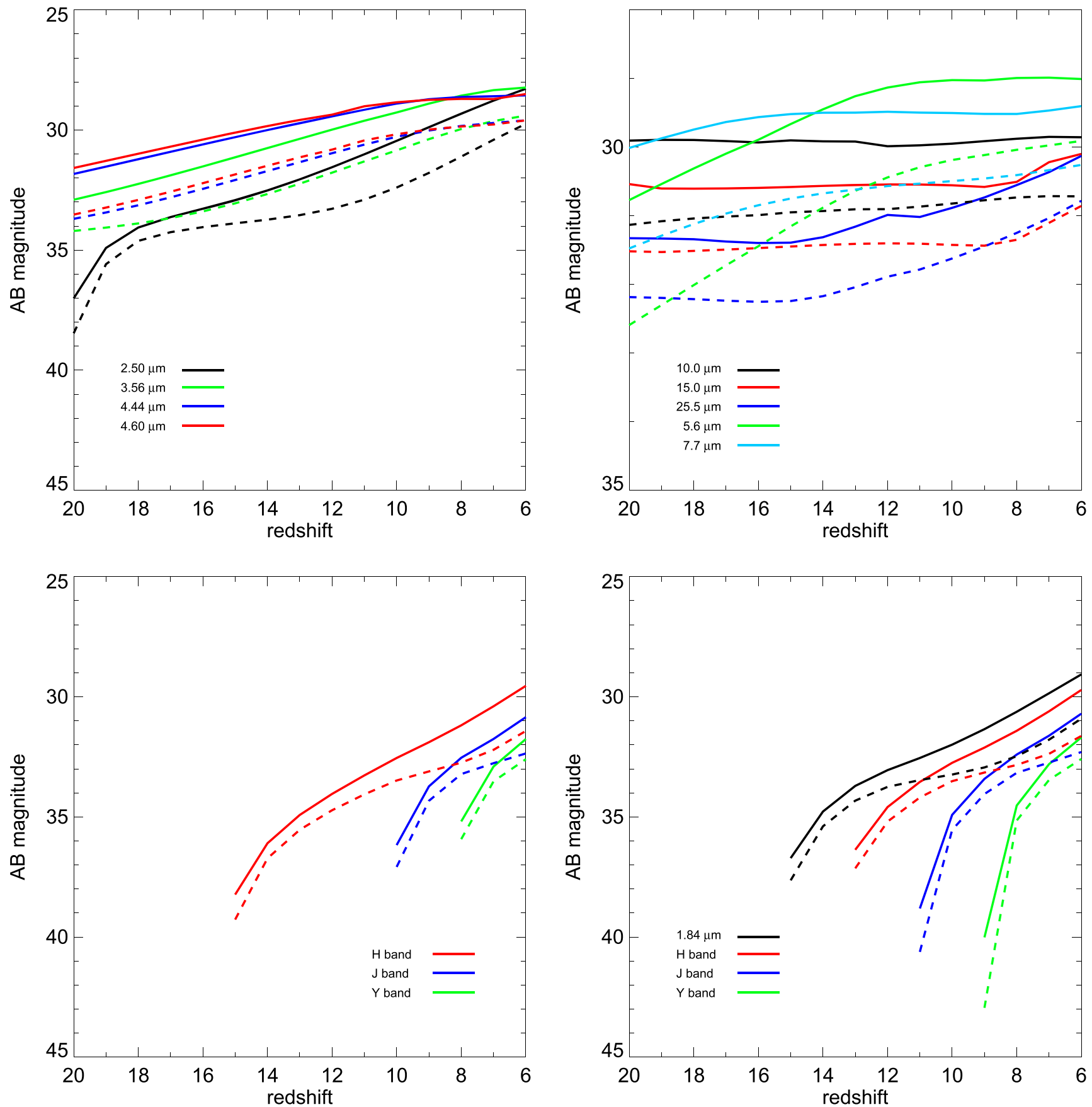

Figure 4. NIR AB magnitudes for the $1.0 M_{\odot} \mathrm{yr}^{-1}$ (solid) and $0.1 M_{\odot} \mathrm{yr}^{-1}$ (dashed) SMSs in JWST, Euclid, and WFIRST bands. Top left: JWST NIRCam bands. Top right: JWST MIRI bands. Bottom left: Euclid. Bottom right: WFIRST.

both missions will enclose thousands of galaxy clusters and massive galaxies, and at $z \sim 6-10$ magnification factors of only 10-100 would be required to reveal either star. It is likely that a sufficient fraction of their survey areas will be lensed to such factors (Rydberg et al. 2018). Even higher magnifications may be possible in future surveys of individual cluster lenses by JWST but at the cost of smaller lensing volumes (Whalen et al. 2013c; Windhorst et al. 2018).

In our Enzo and Cloudy calculations, we have neglected the effect of radiation pressure due to flux from the star on the flows that create it. Modeling these effects in cosmological simulations is challenging in part because they must resolve photospheres on very small scales that preclude the codes from evolving them for long times. Smith et al. (2017a) post-processed simulations of highly resolved atomically cooling halos with $\mathrm{Ly} \alpha$ photon transport and found it could exert mechanical feedback on flows in the vicinity of the star. Radiation hydrodynamical simulations by Luo et al. (2018) and Ardaneh et al. (2018) that neglect resonant $\operatorname{Ly} \alpha$ scattering found that radiation from the protostar in its early stages did not significantly alter flows in its vicinity but did suppress fragmentation, thus promoting the rapid growth of a single supermassive object. In principle, radiation from the SMS could blow out gas and partially expose it to the IGM, but this will have little effect on the $\mathrm{AB}$ magnitudes of the star today because all that would be lost is the mild enhancement of UV flux redward of the Lyman limit by the envelope discussed in Section 3.1. 
While we have only considered cool red supergiant stars, hotter SMSs could be easier to detect because they would produce more flux in the NIR today. Low accretion rates ( $\lesssim 0.005 M_{\odot} \mathrm{yr}^{-1}$; Haemmerlé et al. 2018b) or clumpy accretion due to fragmentation (Sakurai et al. 2015) or turbulence in the disk can produce such stars. However, blue SMS spectra require corrections due to absorption by their atmospheres before the flux that exits the accretion envelope can be calculated. SMSs could also be found at higher redshifts if they exhibit pulsations that temporarily boost their fluxes above the detection limits of the wide-field surveys. Although current stellar evolution codes use implicit solvers and large time steps that do not resolve these oscillations, they can cause the star to periodically brighten and dim by an order of magnitude on timescales of a few weeks in the rest frame. Such variations might also facilitate their detection because their regularity would differentiate them from dusty, red high- $z$ quasars or low- $z$ impostors such as exoplanets. Periodic dimming and brightening could also flag these objects as high- $z$ SMSs in transient surveys proposed for JWST such as FLARE (Wang et al. 2017).

DCBH birth may be the next stage of primordial quasar evolution, and a number of studies have considered their prospects for detection in future NIR surveys. These are also deeply embedded objects in dense, atomically cooled flows and radiative transfer techniques similar to those we have used here are required to model their spectra. One-dimensional radiation hydrodynamics simulations of DCBH emission post-processed with Cloudy have shown that they could be detected by JWST out to $z \sim 20$ (Becerra et al. 2015; Natarajan et al. 2017). We are now post-processing radiation hydrodynamical simulations of the H II region of an SMBH from $z=6-20$ (Smidt et al. 2018) with Cloudy to determine out to what redshifts it could be found by Euclid, WFIRST, and JWST.

We thank the anonymous referee for constructive comments that improved the quality of this paper and Gary Ferland and Peter van Hoof for very helpful discussions on the use of the Cloudy code. D.J.W. was supported by the STFC New Applicant Grant ST/P000509/1, and S.P. was supported by STFC grant ST/N504245/1. T.H. is a JSPS International Research Fellow. E.Z. acknowledges funding from the Swedish National Space Board. S.C.O.G. acknowledges funding from the European Research Council via the ERC Advanced Grant STARLIGHT: Formation of the First Stars (project number 339177) and from the DFG via SFB 881 "The Milky Way System" (sub-projects B1, B2, B8). A.H. was supported by an ARC Future Fellowship (FT120100363), by a Larkins Fellowship from Monash University, by TDLI though a grant from the Science and Technology Commission of Shanghai Municipality (grants No. 16DZ2260200) and National Natural Science Foundation of China (grants No. 11655002). L.H. was sponsored by the Swiss National Science Foundation (project number 200020-172505). The simulations were performed on the Sciama High Performance Compute (HPC) cluster, which is supported by the Institute of Cosmology and Gravitation (ICG), SEPNet and the University of Portsmouth.

\section{ORCID iDs}

Marco Surace (iD https://orcid.org/0000-0002-2723-0098 Daniel J. Whalen (1) https://orcid.org/0000-0001-6646-2337
Tilman Hartwig (ib https://orcid.org/0000-0001-6742-8843

Erik Zackrisson (i) https://orcid.org/0000-0003-1096-2636

S. C. O. Glover (i) https://orcid.org/0000-0001-6708-1317

Samuel Patrick (10 https://orcid.org/0000-0002-5293-699X

Tyrone E. Woods (i) https://orcid.org/0000-0003-1428-5775

Alexander Heger (1D https://orcid.org/0000-0002-3684-1325

\section{References}

Agarwal, B., Smith, B., Glover, S., Natarajan, P., \& Khochfar, S. 2016, MNRAS, 459, 4209

Alvarez, M. A., Wise, J. H., \& Abel, T. 2009, ApJL, 701, L133

Ardaneh, K., Luo, Y., Shlosman, I., et al. 2018, MNRAS, 479, 2277

Bañados, E., Venemans, B. P., Mazzucchelli, C., et al. 2018, Natur, 553, 473

Becerra, F., Greif, T. H., Springel, V., \& Hernquist, L. E. 2015, MNRAS, 446, 2380

Becerra, F., Marinacci, F., Bromm, V., \& Hernquist, L. E. 2018, MNRAS, 480, 5029

Bromm, V., \& Loeb, A. 2003, ApJ, 596, 34

Bryan, G. L., Norman, M. L., O'Shea, B. W., et al. 2014, ApJS, 211, 19

Chen, K.-J., Heger, A., Woosley, S., et al. 2014, ApJ, 790, 162

Ferland, G. J., Chatzikos, M., Guzmán, F., et al. 2017, RMxAA, 53, 385

Freese, K., Ilie, C., Spolyar, D., Valluri, M., \& Bodenheimer, P. 2010, ApJ, 716, 1397

Habouzit, M., Volonteri, M., Latif, M., Dubois, Y., \& Peirani, S. 2016, MNRAS, 463, 529

Haemmerlé, L., Woods, T. E., Klessen, R. S., Heger, A., \& Whalen, D. J. 2018a, ApJL, 853, L3

Haemmerlé, L., Woods, T. E., Klessen, R. S., Heger, A., \& Whalen, D. J. 2018b, MNRAS, 474, 2757

Hartwig, T., Agarwal, B., \& Regan, J. A. 2018, MNRAS, 479, L23

Hirano, S., Hosokawa, T., Yoshida, N., \& Kuiper, R. 2017, Sci, 357, 1375

Hosokawa, T., Yorke, H. W., Inayoshi, K., Omukai, K., \& Yoshida, N. 2013, ApJ, 778, 178

Johnson, J. L., Whalen, D. J., Even, W., et al. 2013a, ApJ, 775, 107

Johnson, J. L., Whalen, D. J., Fryer, C. L., \& Li, H. 2012, ApJ, 750, 66

Johnson, J. L., Whalen, D. J., Li, H., \& Holz, D. E. 2013b, ApJ, 771, 116

Kalirai, J. 2018, ConPh, 59, 251

Latif, M. A., Schleicher, D. R. G., Schmidt, W., \& Niemeyer, J. 2013, MNRAS, 430, 588

Lodato, G., \& Natarajan, P. 2006, MNRAS, 371, 1813

Luo, Y., Ardaneh, K., Shlosman, I., et al. 2018, MNRAS, 476, 3523

Mortlock, D. J., Warren, S. J., Venemans, B. P., et al. 2011, Natur, 474, 616

Natarajan, P., Pacucci, F., Ferrara, A., et al. 2017, ApJ, 838, 117

Planck Collaboration et al. 2016, A\&A, 594, A13

Regan, J. A., \& Haehnelt, M. G. 2009, MNRAS, 396, 343

Rydberg, C.-E., Whalen, D. J., Maturi, M., et al. 2018, arXiv:1805.02662

Sakurai, Y., Hosokawa, T., Yoshida, N., \& Yorke, H. W. 2015, MNRAS, 452,755

Schauer, A. T. P., Regan, J., Glover, S. C. O., \& Klessen, R. S. 2017, MNRAS, 471, 4878

Smidt, J., Whalen, D. J., Johnson, J. L., Surace, M., \& Li, H. 2018, ApJ, 865,126

Smith, A., Becerra, F., Bromm, V., \& Hernquist, L. 2017a, MNRAS, 472, 205 Smith, B. D., Bryan, G. L., Glover, S. C. O., et al. 2017b, MNRAS, 466, 2217 Smith, B. D., Regan, J. A., Downes, T. P., et al. 2018, MNRAS, 480, 3762

Umeda, H., Hosokawa, T., Omukai, K., \& Yoshida, N. 2016, ApJL, 830, L34 Valiante, R., Agarwal, B., Habouzit, M., \& Pezzulli, E. 2017, PASA, 34, e031 Wang, L., Baade, D., Baron, E., et al. 2017, arXiv:1710.07005

Whalen, D., Abel, T., \& Norman, M. L. 2004, ApJ, 610, 14

Whalen, D. J., \& Fryer, C. L. 2012, ApJL, 756, L19

Whalen, D. J., Fryer, C. L., Holz, D. E., et al. 2013a, ApJL, 762, L6

Whalen, D. J., Johnson, J. L., Smidt, J., et al. 2013b, ApJ, 777, 99

Whalen, D. J., Smidt, J., Even, W., et al. 2014, ApJ, 781, 106

Whalen, D. J., Smidt, J., Johnson, J. L., et al. 2013c, arXiv:1312.6330

Windhorst, R. A., Timmes, F. X., Wyithe, J. S. B., et al. 2018, ApJS, 234, 41

Wise, J. H., Turk, M. J., \& Abel, T. 2008, ApJ, 682, 745

Woods, T. E., Bhaskar, A., Volker, B., et al. 2018, arXiv:1810.12310

Woods, T. E., Heger, A., Whalen, D. J., Haemmerlé, L., \& Klessen, R. S. 2017, ApJL, 842, L6

Zackrisson, E., Scott, P., Rydberg, C.-E., et al. 2010a, ApJ, 717, 257

Zackrisson, E., Scott, P., Rydberg, C.-E., et al. 2010b, MNRAS, 407, L74 